\title{
In Vitro-In Silico Comparison of Pulsed Oxygen Delivery From Portable Oxygen Concentrators Versus Continuous Flow Oxygen Delivery
}

\author{
John Z Chen, Ira M Katz, Marine Pichelin, Kaixian Zhu, Georges Caillibotte, Warren H Finlay, \\ and Andrew R Martin
}

\begin{abstract}
BACKGROUND: Portable oxygen concentrators (POCs) deliver oxygen in intermittent pulses. The challenge of establishing equivalence between continuous flow oxygen and nominal pulse flow settings on different POCs is well known. In vitro bench measurements and in silico mathematical modeling were used to compare the performance of 4 POCs versus continuous flow oxygen by predicting the $\mathrm{F}_{\mathrm{IO}_{2}}$ at the trachea and entering the acini. METHODS: Each of the 4 POCs was connected to a 3-dimensional printed replica of a human adult nasal airway via nasal cannula. A test lung simulated 3 breathing patterns representative of a patient with COPD at rest, during exercise, and while asleep. POCs were tested for each breathing pattern at all integer pulse flow settings. Volume-averaged $\mathrm{F}_{\mathrm{IO}_{2}}$ was calculated by analyzing oxygen concentrations and inhalation flow over time. In vitro oxygen waveforms were then combined with a single-path mathematical model of the lungs to assess oxygen transport through the conducting airways. In vitro experiments and mathematical modeling were repeated for continuous flow oxygen. RESULTS: Continuous flow oxygen consistently delivered more ( $>2 \%$ absolute) oxygen in terms of volume-averaged $\mathrm{F}_{\mathrm{IO}_{2}}$ for all nominally equivalent pulse flow settings of $>2$. Differences were also observed when comparing performances between different POCs, particularly at high device settings (5 and 6). Simulations showed that efficiency of delivery to the acinar region of the lungs was higher in pulse flow than in continuous flow oxygen but that continuous flow oxygen generally delivered a higher absolute volume of oxygen. Differences in absolute oxygen delivery per breath between continuous flow oxygen and pulse flow were smaller for acinar delivery than for tracheal delivery. CONCLUSIONS: Significant differences in $\mathrm{POC}$ performance based on volume-averaged $\mathrm{F}_{\mathrm{IO}_{2}}$ were found between pulse flow and continuous flow oxygen, and among pulse flow modes in different POCs. Although pulse flow was a more efficient mode of delivery than continuous flow oxygen, continuous flow oxygen delivered a greater absolute volume of oxygen per breath. Key words: long-term oxygen therapy (LTOT); ambulatory oxygen; portable oxygen concentrator (POC); lung simulator; nasal cannula; chronic obstructive pulmonary disease; oxygen therapy; lung model; trumpet model; pulse. [Respir Care 2019;64(2):117-129. (c) 2019 Daedalus Enterprises]
\end{abstract}

\section{Introduction}

Long-term oxygen therapy has been shown to prolong life in patients with COPD and severe daytime hypox-

Mr Chen, Dr Finlay, and Dr Martin are affiliated with the Department of Mechanical Engineering, University of Alberta, Edmonton, Alberta, Canada. Drs Katz, Pichelin, and Caillibotte are affiliated with the Medical R\&D, Air Liquide Santé International, Centre de Recherche Paris-Sa- emia. ${ }^{1,2}$ Oxygen has historically been provided as a continuous flow supplied to the patient interface, but, more recently, intermittent delivery methods triggered by a patient's breathing have been developed as portable or

\footnotetext{
clay, Les Loges-en-Josas, France. Dr Zhu is affiliated with Centre Explor!, Air Liquide Healthcare, Gentilly, France.

This study was performed at the University of Alberta in Edmonton, Alberta, Canada.
} 
cost-saving alternatives. ${ }^{3-5}$ Portable oxygen concentrators (POCs) are the latest class of devices in the intermittent delivery paradigm. ${ }^{3,6}$ Because these devices concentrate existing atmospheric oxygen, they do not

See the Related Editorial on Page 230

require a stationary oxygen source and provide a portable option for supplemental oxygen therapy. Although some POCs can deliver oxygen continuously at limited flows, many recent-generation devices deliver oxygen exclusively by using pulse flow, in which a short-duration flow, or pulse, of oxygen is provided only when patient inspiration is detected.7 A recent study showed that subjects with COPD who required long-term oxygen therapy generally preferred a single-source POC instead of a combined stationary and portable oxygen source, citing the practicality of the system as its main advantage. ${ }^{8}$ However, the same study showed that insufficient oxygenation $\left(\mathrm{S}_{\mathrm{pO}_{2}}<90 \%\right)$ was more frequent among users of single-source POC.

The challenge of establishing equivalence between continuous flow oxygen and nominal pulse flow device settings on different devices is well known ${ }^{5,9}$ and provides motivation for the development of physiologically representative in vitro testing methods. Chen et al ${ }^{10}$ recently outlined a methodology to compare pulse flow oxygen delivery from a commercially available POC with continuous flow oxygen delivery from a stationary cylinder by using a set of 15 realistic airway replicas. Use of these replicas, together with a lung simulator in in vitro experiments allowed for precise control of simulated breathing parameters in anatomically representative models of the upper airways and made it possible to account for potential intersubject variability due to variance in airway geometries as well as allowing modes of

Drs Katz, Pichelin, Zhu, and Caillibotte are current employees of Air Liquide, a major provider of home oxygen therapy. The authors report no other conflicts of interest in this work.

This work was funded by Air Liquide and by Alberta Economic Development and Trade, and benefited from an equipment grant from the Canadian Natural Sciences and Engineering Research Council. Portable oxygen concentrators were supplied for testing by VitalAire Canada and by Inogen.

Supplementary material related to this paper is available at http:// www.rcjournal.com.

Correspondence: John Z Chen, 2-9 Mechanical Engineering Building, University of Alberta, Edmonton, AB T6G 2G8, Canada. E-mail: jzc@ualberta.ca.

DOI: $10.4187 /$ respcare. 06359

\section{QUICK LOOK}

\section{Current knowledge}

Long-term oxygen therapy prolongs life in patients with COPD and severe daytime hypoxemia. Portable oxygen concentrators (POCs) that deliver pulsed oxygen intermittently are purported to be more oxygenefficient than continuous flow oxygen. Performance differences between pulse flow and continuous flow oxygen and among different POCs operated at the same numerical setting have been reported.

\section{What this paper contributes to our knowledge}

$\mathrm{F}_{\mathrm{IO}_{2}}$ at the trachea, assessed in vitro in a benchtop model that incorporated a realistic upper airway replica and simulated breathing, was higher for continuous flow oxygen compared with pulse flow at nominally equivalent flows and/or POC numerical settings. Differences in $\mathrm{F}_{\mathrm{IO}_{2}}$ were also observed among the different POCs operated at the same numerical setting. When the in vitro data were combined with in silico modeling, it was predicted that pulse flow, compared with continuous flow oxygen, delivers oxygen more efficiently to the acinar region of the lung. However, for the POCs evaluated, the absolute volume of oxygen delivered to the acini per breath was predicted to be greater for continuous flow oxygen than for pulse flow across the majority of cases studied.

failure to be assessed when a POC failed to detect an inspiratory effort.

By measuring the real-time oxygen concentration at the airway replica outlet (representative of the trachea) during inspiration, a volume-averaged $\mathrm{F}_{\mathrm{IO}_{2}}$ was obtained that represented the fraction of oxygen contained in a given inhaled tidal volume $\left(\mathrm{V}_{\mathrm{T}}\right) \cdot{ }^{10}$ In other words, these volumeaveraged $\mathrm{F}_{\mathrm{IO}_{2}}$ values represent the ratios between the total volume of inhaled oxygen (including both supplemental oxygen and oxygen in the entrained air) and the inhaled $\mathrm{V}_{\mathrm{T}}$, and provide a common basis for comparison between pulse flow and continuous flow oxygen. ${ }^{10}$

In building on this recent work, the present study had 2 primary objectives. The first was to compare the performance of several POCs against each other and against continuous flow oxygen by using volume-averaged $\mathrm{F}_{\mathrm{IO}_{2}}$ at the trachea as a measure of oxygen delivery. The second objective was to characterize the transport of oxygen pulses from the trachea through the conducting airways via mathematical modeling. This enabled the assessment of the impact of continuous flow oxygen flows, pulse flow settings, and breathing parameters on transport of oxygen 


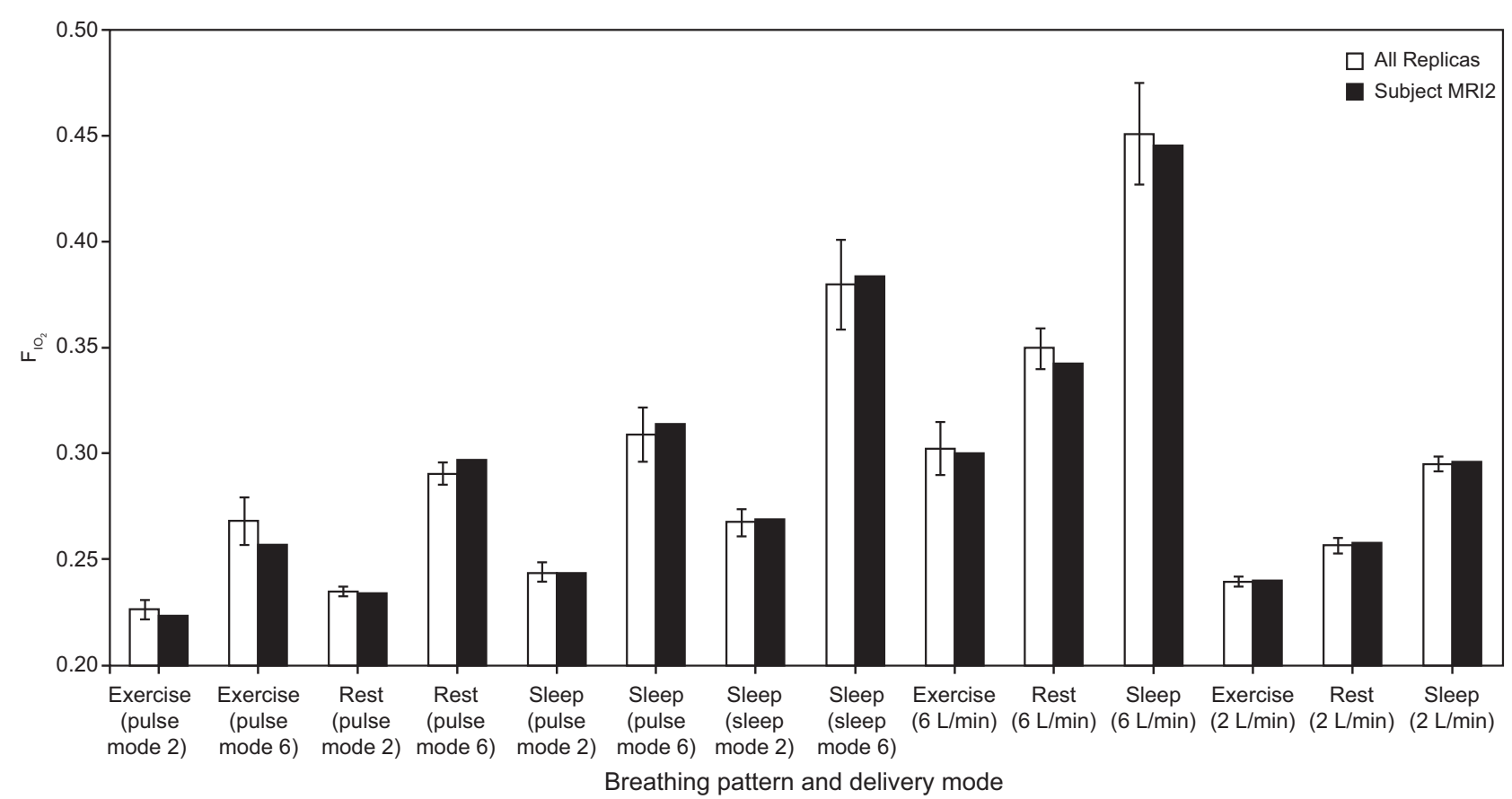

Fig. 1. Comparison of volume-averaged $\mathrm{F}_{\mathrm{IO}_{2}}$ by using Subject $\mathrm{MRI}$, with a mean volume-averaged $\mathrm{F}_{\mathrm{IO}_{2}}$ across 15 airway replicas. Data for both pulse deliveries from a SimplyGo portable oxygen concentrator and continuous flow oxygen from a stationary source of compressed oxygen are included. Error bars indicate $\pm 1 \mathrm{SD}$.

through the conducting airways to the gas-exchange region of the lung.

\section{Methodology}

\section{Selection of a Representative Airway Replica}

In Chen et al, ${ }^{10}$ testing was limited to a single POC evaluated at 2 integer pulse settings, one high (6) and one low (2). It was found that intersubject variability among 15 airway geometries had only a small $(<5 \%$ coefficient of variation) impact on volume-averaged $\mathrm{F}_{\mathrm{IO}_{2}}$ values. ${ }^{10}$ Therefore, it was deemed reasonable to use only a single representative replica for comparative testing in the present work. A single replica was selected on the criterion that the volume-averaged $\mathrm{F}_{\mathrm{IO}_{2}}$ value obtained by using this replica (for either continuous flow oxygen or pulse flow) was closest to the average value obtained across the set of 15 replicas. Volume-averaged $\mathrm{F}_{\mathrm{IO}_{2}}$ values obtained for the selected airway replica (Subject MRI2) are compared in Figure 1, with average and standard variation of values obtained across all replicas. The selected replica had a total interior volume of $44.6 \mathrm{~mL}$ and an interior surface area of $287 \mathrm{~cm}^{2}$. These values were obtained by using MeshLab (Visual Computing Laboratory, Istituto di Scienza e Tecnologie dell'Informazione, Pisa, Italy) and ParaView (Kitware, Clifton Park, New York).

\section{Airway Experiments}

Experiments were performed by using the experimental apparatus described in Chen et al. ${ }^{10}$ The test set of POCs consisted of a SimplyGo (Philips Respironics, Murrysville, Pennsylvania), a SimplyGo Mini (Philips Respironics, Murrysville, Pennsylvania), a One G3 (Inogen, Goleta, California), and a One G4 (Inogen, Goleta, California). A photograph of the tested POCs, the weight of each device, and a visual comparison of device sizes are shown in Figure 2. Specifications for each device are shown in Table 1 . To account for the variety of use conditions that a patient with COPD may experience in his or her everyday life and to explore the effect of varying breathing frequencies and $\mathrm{V}_{\mathrm{T}}$ on POC performance and $\mathrm{F}_{\mathrm{IO}_{2}}$, 3 different breathing patterns were chosen, representative of a patient with COPD at rest, while asleep, and during light exercise.

The inspiration and expiration flow waveforms were each modeled by using a half-sinusoid and actuated by using a lung simulator (ASL 5000 Breathing Simulator, IngMar Medical, Pittsburgh, Pennsylvania). A schematic of the experimental apparatus is shown in Figure 3. Breathing parameters for each of these patterns (at rest, while asleep, and during light exercise) are provided in Table 2 . The rest and exercise breathing parameters were chosen based on average values reported previously by 


\section{Pulsed Dose vs Continuous Flow Oxygen Delivery}

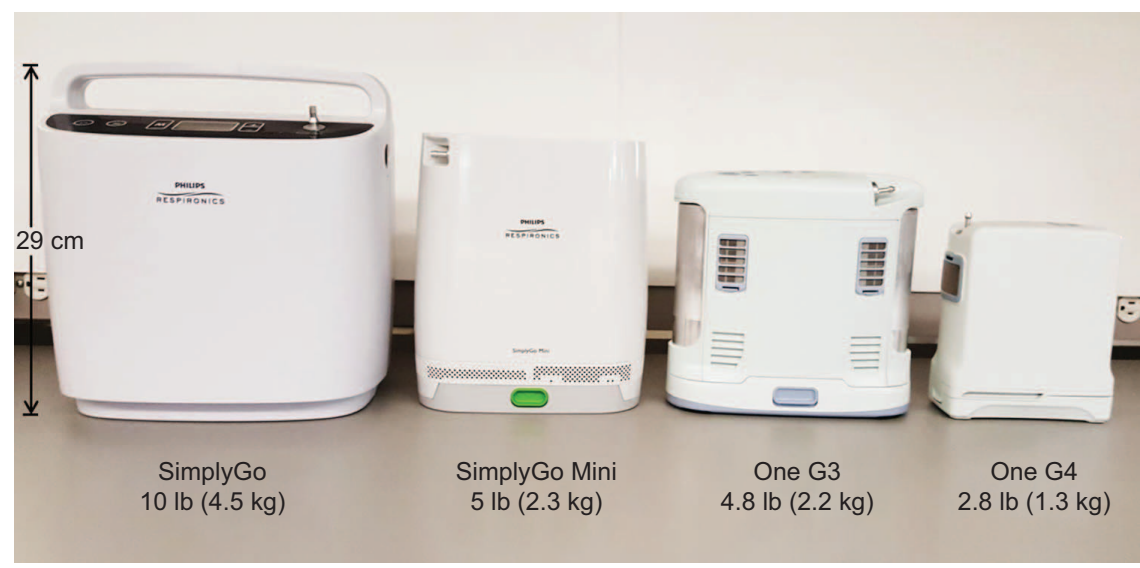

Fig. 2. Commercial portable oxygen concentrators tested in this study.

Table 1. Specifications for Portable Oxygen Concentrators Used in This study

\begin{tabular}{llccc}
\hline \hline \multicolumn{1}{c}{ Device } & \multicolumn{1}{c}{ Vendor } & Weight (lb) & $\begin{array}{c}\text { Dimensions, length } \times \\
\text { height } \times \text { width (cm) }\end{array}$ & $\begin{array}{c}\text { Pulse Flow Nominal Device } \\
\text { Settings (arbitrary units) }\end{array}$ \\
\hline SimplyGo & Philips Respironics & 10.0 & $29.2 \times 25.4 \times 15.2$ & $1-6$ \\
SimplyGo Mini & Philips Respironics & 5.0 & $23.9 \times 21.1 \times 9.1$ & $1-5$ \\
One G3 & Inogen & 4.8 & $22.2 \times 21 \times 7.6$ & $1-5$ \\
One G4 & Inogen & 2.8 & $15.01 \times 18.3 \times 6.8$ & $1-3$ \\
\hline
\end{tabular}

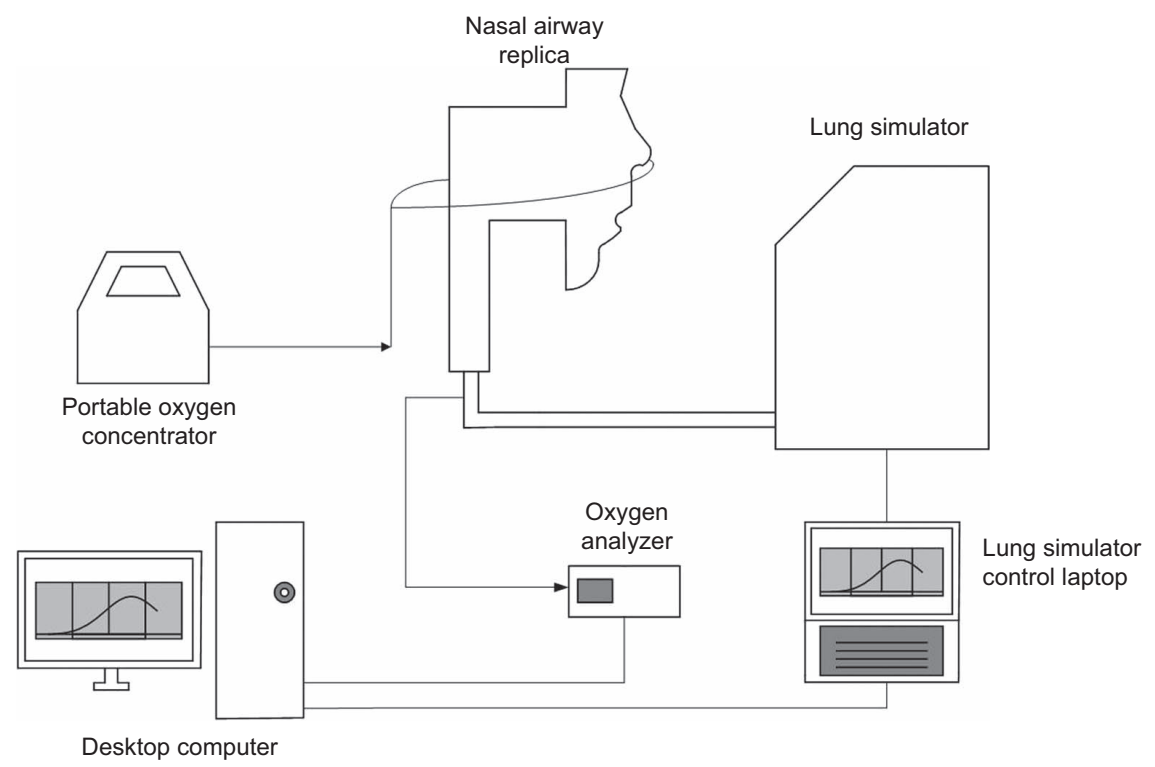

Fig. 3. Schematic of apparatus used in experiments that involve airway replicas. Arrows indicate direction of oxygen flow. From Reference 10 , with permission.

Chatila et $\mathrm{al}^{11}$ for 10 subjects with COPD at baseline (rest) and while on continuous flow oxygen $(2.5-6 \mathrm{~L} / \mathrm{min})$ while performing light exercise on a cycle ergometer. Parameters for the sleep breathing pattern were chosen based on average values measured by Hudgel et al ${ }^{12}$ for 13 subjects with COPD while asleep.

\section{Calculation of Volume-Averaged Tracheal $\mathrm{F}_{\mathrm{IO}_{2}}$}

Example flow and oxygen fraction waveforms are shown in Figure 4. The flow of oxygen passing through the trachea over time was calculated by multiplying inspiration flow with measured oxygen concentrations at the same 
Table 2. Breathing Parameters of Representative Breathing Profiles for Patients With COPD at Rest, Doing Light Exercise, and While Asleep

\begin{tabular}{lccc}
\hline \hline \multicolumn{1}{c}{ Parameter } & Rest & Exercise & Sleep \\
\hline Tidal volume, $\mathrm{mL}$ & 640 & 800 & 520 \\
Inspiratory time, s & 1.20 & 0.96 & 1.79 \\
Expiratory time, $\mathrm{s}$ & 2.33 & 1.77 & 2.93 \\
Breathing frequency, breaths/min & 17 & 22 & 13 \\
\hline
\end{tabular}

point in time. The beginning and the end of inspiration were identified as times when oxygen flow crossed $0 \mathrm{~mL} / \mathrm{s}$. These oxygen flows were then numerically integrated via the trapezoidal rule from the start to the end of inspiration to determine a volume of oxygen inspired for that breath. Finally, volume-averaged $\mathrm{F}_{\mathrm{IO}_{2}}$ was obtained by dividing the inspired volume of oxygen by $\mathrm{V}_{\mathrm{T}} . \mathrm{F}_{\mathrm{IO}_{2}}$ for each combination of device, device setting, and breathing pattern was taken as the average of 5 consecutive breaths after a steady state in the end-expiratory oxygen concentration was observed. The variability among $\mathrm{F}_{\mathrm{IO}_{2}}$ values obtained in experiments repeated on separate days was found in preliminary testing to be of similar magnitude as variability between individual breaths. One-way analysis of variance was performed to compare the differences in volumeaveraged tracheal $\mathrm{F}_{\mathrm{IO}_{2}}$ by analyzing the simple main effects of device setting-continuous flow oxygen flow and mode of delivery (4 POCs and continuous flow oxygen). Multiple post hoc comparisons were then done by using the Tukey test, with $P<.05$ considered significant.

\section{Measurement of Pulse Characteristics}

An $\mathrm{O}_{2}$ Conserver Testing System (1,130 series, Hans Rudolph, Shawnee, Kansas) was used to obtain oxygen pulse volumes, durations, and delays for each setting and each POC. POCs were connected to the testing system by using standard oxygen tubing. Data were recorded as the average of 20 successive pulses for each breathing pattern-setting combination. Average pulse characteristics were calculated only from breaths when the device was properly triggered.

\section{Prediction of Pulse Flow Volume-Averaged $\mathrm{F}_{\mathrm{IO}_{2}}$}

Chen et al ${ }^{10}$ previously published an algebraic model that predicts in vitro volume-averaged $\mathrm{F}_{\mathrm{IO}_{2}}$ in realistic airway replicas based on pulse characteristics. The model uses airway replica internal volume, measured pulse volumes, ambient oxygen concentration, and pulse oxygen concentration to first calculate an internal oxygen concentration in the chamber of the test lung or, equivalently, the amount of oxygen passing the entrance to the chamber of the test lung:

$$
\begin{aligned}
X_{O_{2}, c}=X_{O_{2, \text { ambient }}}+V_{\text {pulse }} & \left(X_{O_{2, \text { pulse }}}\right. \\
& \left.-X_{O_{2, \text { ambient }}}\right) /\left(V_{T}-V_{A W}\right)
\end{aligned}
$$

where $X_{O_{2}, c}$ is the test lung chamber oxygen fraction, $X_{O_{2} \text { ambient }}$ is the fraction of oxygen in ambient air, $V_{\text {pulse }}$ is the volume of the oxygen pulse, $V_{T}$ is the tidal volume, $V_{A W}$ is the total volume of the airways (both upper and lower respiratory tracts), and $X_{\mathrm{O}_{2} \text {, pulse }}$ is the oxygen fraction ( 0.94 for the Philips devices, 0.95 for the Inogen devices) of the pulse.

In vitro volume-averaged $\mathrm{F}_{\mathrm{IO}_{2}}$ is then predicted by using the following equation:

$$
\begin{aligned}
F_{I O_{2}}=\left(V_{\text {pulse }}\right. & X_{O_{2,} \text { pulse }}+V_{\text {replica }} X_{O_{2, c}} \\
& \left.+\left(V_{T}-V_{\text {replica }}-V_{\text {pulse }}\right) X_{O_{2, \text { ambient }}}\right) / V_{T}
\end{aligned}
$$

\section{Mathematical Modeling of Oxygen Transport Through the Conducting Airways}

Oxygen flowing past the trachea enters the conducting airways and may eventually be transported to alveolar regions of the lung where gas exchange occurs. To describe the transport of the oxygen pulse through the conducting airways to the acini, a modified version of a mathematical model previously described by Martin et al ${ }^{13}$ for assessing nitric oxide transport and uptake was used. The model, written in MATLAB (Mathworks, Natick, Massachusetts), assumes a simplified single-path, single-alveolar compartment lung structure, with no oxygen exchange occurring in the conducting airways.

By using oxygen concentration waveforms measured at the trachea over time from the in vitro measurements described above as a boundary condition, the present model simulated the transport of oxygen to the acini by a combination of bulk convection and axial dispersion through the conducting tracheobronchial airways, which were modeled as a series of branching tubes whose dimensions become smaller with each branching generation. The mathematical model assumed these tubes to be rigid, cylindrical, and bifurcating between each airway generation. Baseline dimensions were taken from an adult airway model provided by Finlay et al ${ }^{14}$ based on airway data from Phillips et a ${ }^{15}$ for an adult with a functional residual capacity of $3000 \mathrm{~mL}$ (Table 3). Additional details that pertain to the mathematical model are described in the supplementary material (see the supplementary materials at http://www.rcjournal.com).

Inputs to the model consisted of the following:

1. Comma-separated value (.csv) versions of individual oxygen concentration waveforms over the course of inhalation (one per breathing pattern-device setting combination), which were extracted manually from raw oxygen concentration data. 


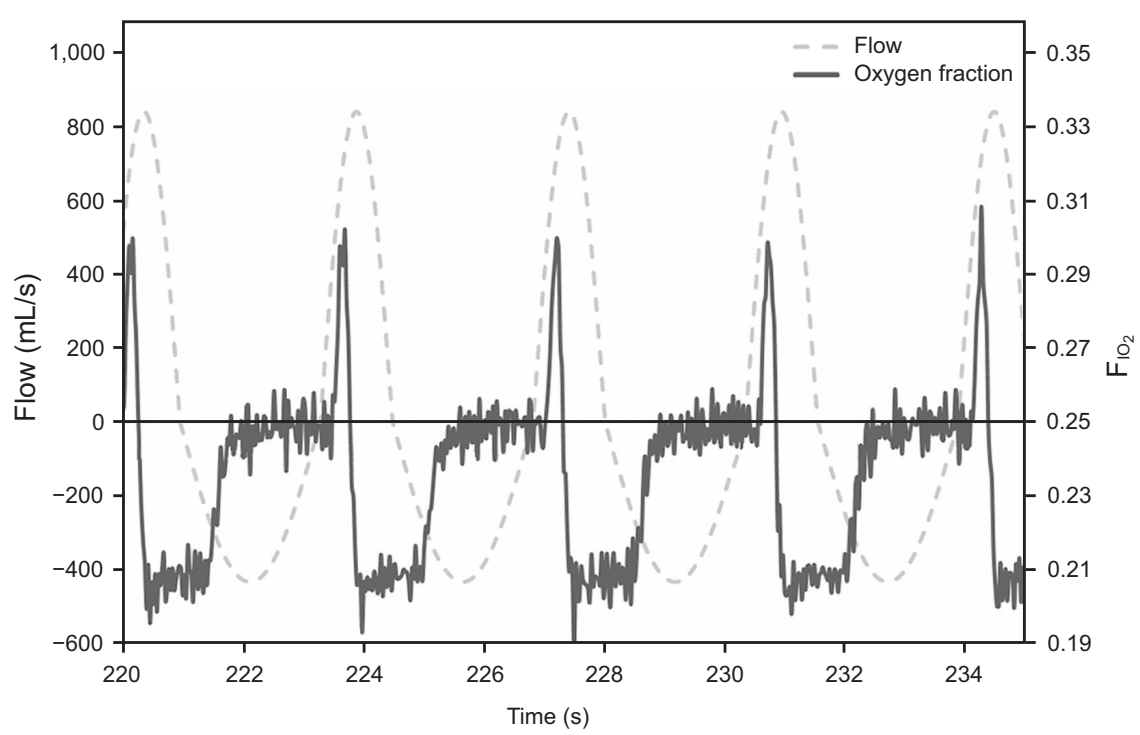

Fig. 4. Sample flow and oxygen fraction waveforms generated by the experimental apparatus for pulsed delivery of oxygen. From Reference 10 , with permission.

Table 3. Cylindrical Airway Dimensions of Tracheobronchial Airways at Each Generation in the Lung Model

\begin{tabular}{lcc}
\hline \hline \multirow{2}{*}{ Generation No. } & \multicolumn{2}{c}{ Airway Dimensions } \\
\cline { 2 - 3 } & Diameter $(\mathrm{cm})$ & Length $(\mathrm{cm})$ \\
\hline 0 & 1.96 & 13.53 \\
1 & 1.53 & 3.92 \\
2 & 1.22 & 3.11 \\
3 & 0.97 & 2.48 \\
4 & 0.77 & 1.93 \\
5 & 0.62 & 1.23 \\
6 & 0.49 & 0.98 \\
7 & 0.39 & 0.90 \\
8 & 0.32 & 0.81 \\
9 & 0.24 & 0.71 \\
10 & 0.17 & 0.61 \\
11 & 0.13 & 0.49 \\
12 & 0.10 & 0.39 \\
13 & 0.08 & 0.30 \\
14 & 0.07 & 0.24 \\
\hline
\end{tabular}

2. Simulation breathing parameters, including $\mathrm{V}_{\mathrm{T}}$, breathing frequency and the ratio of inhalation to exhalation time. Values of inhalation to exhalation ratio were 0.515 for the exercise breathing pattern, 0.538 for the rest breathing pattern, and 0.613 for the sleep breathing pattern. These were used to generate the same idealized breathing patterns as those used in the in vitro experiments.

3. Pre-inhalation oxygen concentration in the conducting airways. Because the present analysis considered only the transport of oxygen through conducting airways during a single inhalation and not uptake of oxygen to the blood, the oxygen concentration throughout the conducting airways was set to zero at the beginning of each simulation. Under this condition, only the transport of oxygen freshly inhaled through the upper airway was considered.

Outputs of the model included time-varying oxygen concentrations at individual airway generations distal to the trachea, the total volume of oxygen delivered to the acini, and the ratio between the oxygen volume delivered to the acini and that delivered to the trachea, which represented acinar delivery efficiency. Because the initial concentration of oxygen in the conducting airways was set to zero at the start of inhalation, the acinar delivery efficiency can be viewed as the fraction of oxygen passing the trachea that also passes into the acini during an inhalation. Based on preliminary simulations, the coefficient of variation in delivered oxygen volume between simulations performed for different individual breaths ranged from approximately 0.1 to $4 \%$ of the mean. Therefore, it was sufficient to use only a single breath from each breathing pattern-device-pulse setting combination in the model calculations.

\section{Results}

\section{Comparisons of POC Performance}

The comparisons of volume-averaged $\mathrm{F}_{\mathrm{IO}}$, pulse volume, and pulse duration for continuous flow oxygen from a compressed oxygen source versus pulse flow from each of the POCs are shown in Figure 5. The differences between each device, by showing pulse flow 


\section{Pulsed Dose vs Continuous Flow Oxygen Delivery}
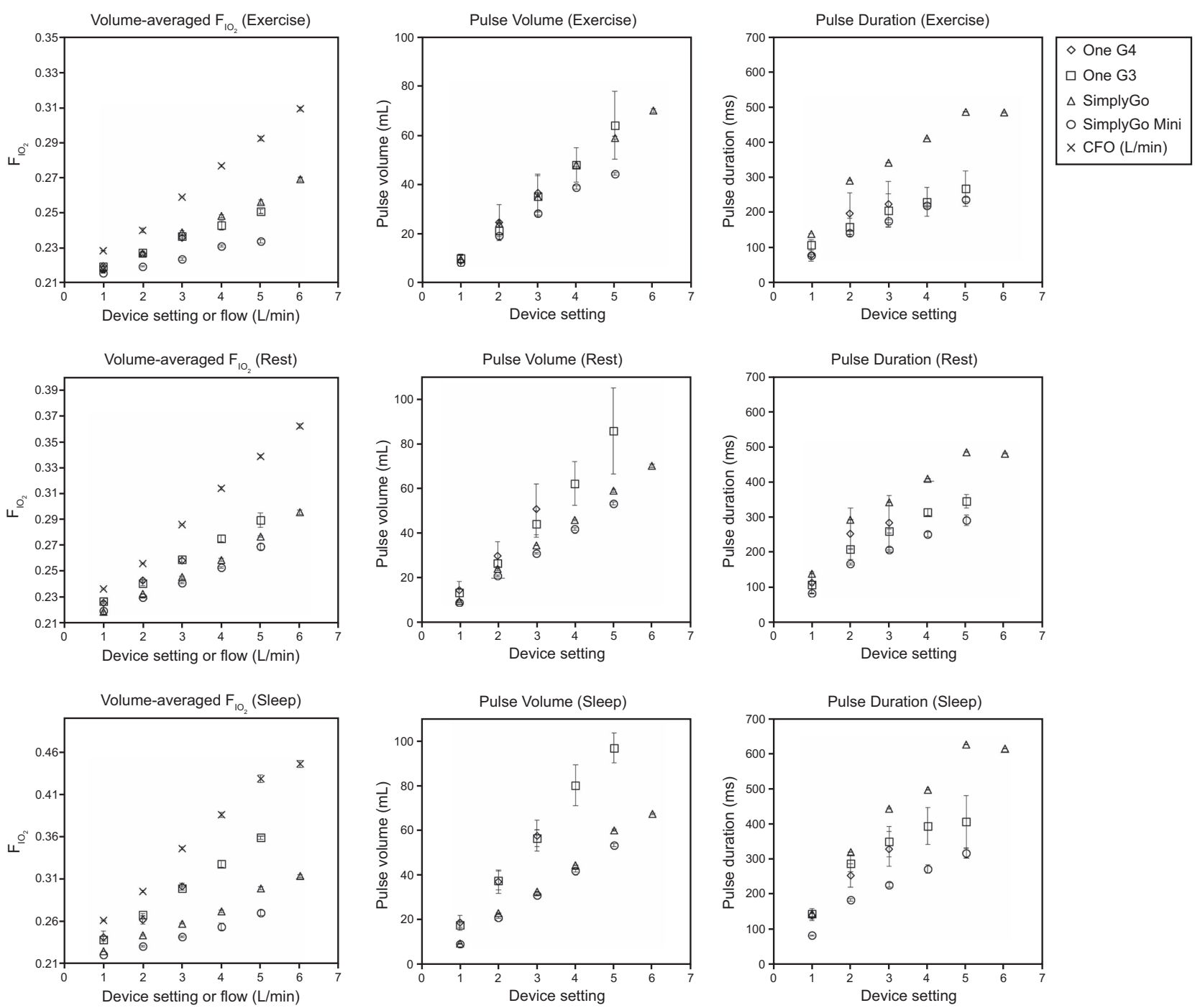

Fig. 5. Volume-averaged $\mathrm{F}_{1 \mathrm{O}_{2}}$ and pulse characteristics for each of the tested portable oxygen concentrators and continuous flow oxygen across 3 breathing patterns. $\mathrm{F}_{\mathrm{IO}_{2}}$ values were averaged over 5 consecutive breaths. Other pulse characteristics averaged $>20$ consecutive breaths. Error bars indicate $\pm 1 \mathrm{SD}$.

profiles over time at pulse setting 2 for each of the POCs, are illustrated in Figure 6.

Statistical analysis showed that, when the mode of delivery was held constant, there were statistically significant differences $(P<.001$ in every case $)$ among all device settings-continuous flow oxygen flows for each mode of delivery in all the breathing patterns. With device settingcontinuous flow oxygen held constant, several homogeneous subgroups (groups of delivery modes with statistically similar performance) emerged under post hoc analysis, which are listed in Table 4.

Due to the high repeatability of the in vitro test methods used in some cases, very small differences in $\mathrm{F}_{\mathrm{IO}_{2}}$ were statistically significant. Therefore, in addition to statistical significance, a threshold for a practical or anticipated clinically important difference in $\mathrm{F}_{\mathrm{IO}_{2}}$ was defined to be $>2 \%$ (abso- lute percentage oxygen) when following Zhou and Chatburn. ${ }^{16}$ By using this more-demanding threshold, continuous flow oxygen still delivered a significantly higher $\mathrm{F}_{\mathrm{IO}_{2}}$ than pulse flow in at least one of the devices at all nominally equivalent device settings of $\geq 2$. The magnitude of this difference decreased as minute volume (which in our model included an increase in both $\mathrm{V}_{\mathrm{T}}$ and breathing frequency) increased.

For the 2 Philips devices, the SimplyGo consistently delivered more oxygen than the SimplyGo Mini, although $\mathrm{F}_{\mathrm{IO}_{2}}$ values were within 2\% (absolute) for the majority of breathing pattern-setting number combinations, with the exception of setting 5 in the sleep and exercise breathing patterns. For the Inogen devices, no anticipated clinically important $\mathrm{F}_{\mathrm{IO}_{2}}$ difference was 


\section{Pulsed Dose va Continuous Flow Oxygen Delivery}

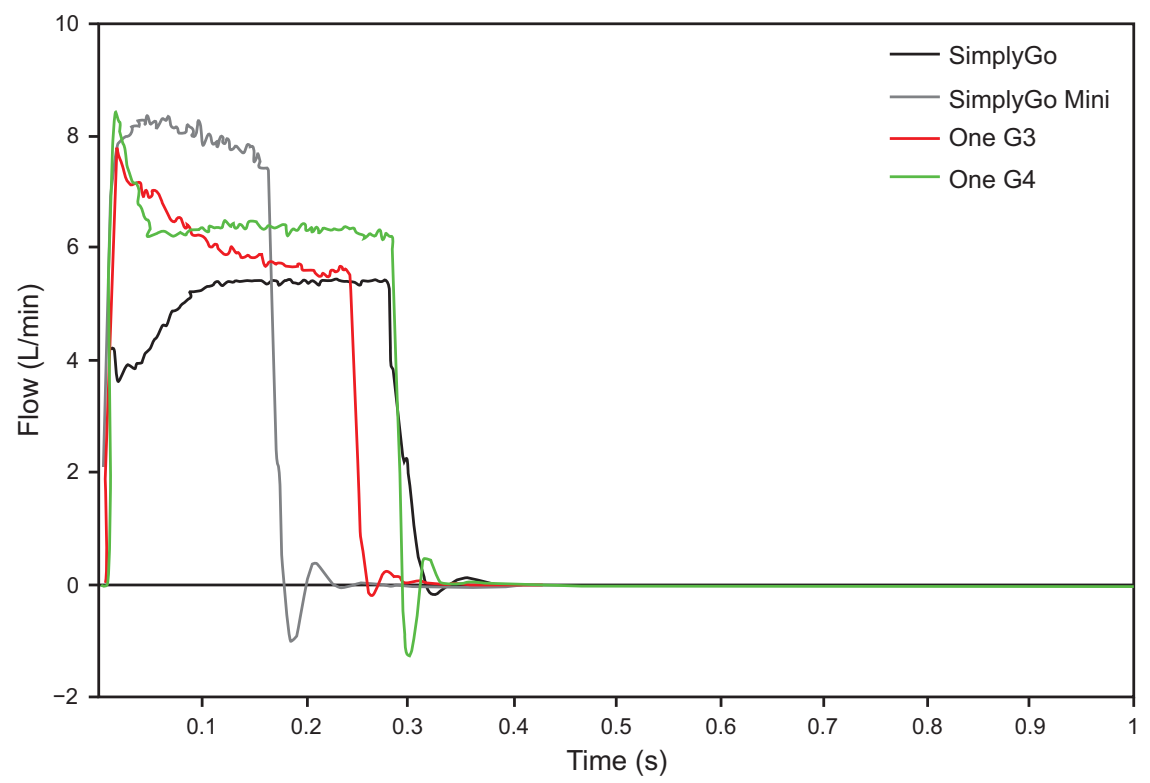

Fig. 6. Pulse flow curves generated by the $\mathrm{O}_{2}$ Conserver Testing System for each portable oxygen concentrators (POC) at setting 2 for each device and breathing frequency of 17 breaths $/ \mathrm{min}$.

Table 4. Subgroups With Statistically Similar Volume-Averaged Tracheal $\mathrm{F}_{\mathrm{IO}_{2}}(P>.05)$ Segregated by Device Setting, CFO, and Breathing Pattern

\begin{tabular}{|c|c|c|c|c|c|c|}
\hline \multirow[b]{2}{*}{ Breathing Pattern } & \multicolumn{6}{|c|}{ Device setting (arbitrary units) or CFO (L/min) } \\
\hline & 1 & 2 & 3 & 4 & 5 & 6 \\
\hline \multirow[t]{4}{*}{ Exercise } & $\mathrm{CFO}$ & $\mathrm{CFO}$ & $\mathrm{CFO}$ & $\mathrm{CFO}$ & $\mathrm{CFO}$ & $\mathrm{CFO}$ \\
\hline & SimplyGo & SimplyGo & SimplyGo & SimplyGo & SimplyGo & \multirow[t]{3}{*}{ SimplyGo } \\
\hline & $\begin{array}{l}\text { One G3 } \\
\text { One G4 }\end{array}$ & $\begin{array}{l}\text { One G3 } \\
\text { One G4 }\end{array}$ & $\begin{array}{l}\text { One G3 } \\
\text { One G4 }\end{array}$ & One G3 & One G3 & \\
\hline & SimplyGo Mini & SimplyGo Mini & SimplyGo Mini & SimplyGo Mini & SimplyGo Mini & \\
\hline \multirow[t]{5}{*}{ Rest } & $\mathrm{CFO}$ & $\mathrm{CFO}$ & $\mathrm{CFO}$ & $\mathrm{CFO}$ & $\mathrm{CFO}$ & $\mathrm{CFO}$ \\
\hline & One G3 & One G4 & One G3 & One G3 & One G3 & \multirow[t]{4}{*}{ SimplyGo } \\
\hline & One G4 & One G3 & One G4 & SimplyGo & SimplyGo & \\
\hline & \multirow{2}{*}{$\begin{array}{l}\text { SimplyGo } \\
\text { SimplyGo Mini }\end{array}$} & SimplyGo & SimplyGo & & & \\
\hline & & SimplyGo Mini & SimplyGo Mini & SimplyGo Mini & SimplyGo Mini & \\
\hline \multirow[t]{4}{*}{ Sleep } & $\mathrm{CFO}$ & $\mathrm{CFO}$ & $\mathrm{CFO}$ & $\mathrm{CFO}$ & $\mathrm{CFO}$ & $\mathrm{CFO}$ \\
\hline & \multirow{2}{*}{$\begin{array}{l}\text { One G3 } \\
\text { One G4 }\end{array}$} & One G4 & One G3 & One G3 & One G3 & \multirow[t]{3}{*}{ SimplyGo } \\
\hline & & One G3 & One G4 & & SimplyGo & \\
\hline & $\begin{array}{l}\text { SimplyGo } \\
\text { SimplyGo Mini }\end{array}$ & $\begin{array}{l}\text { SimplyGo } \\
\text { SimplyGo Mini }\end{array}$ & $\begin{array}{l}\text { SimplyGo } \\
\text { SimplyGo Mini }\end{array}$ & $\begin{array}{l}\text { SimplyGo } \\
\text { SimplyGo Mini }\end{array}$ & SimplyGo Mini & \\
\hline
\end{tabular}

For each breathing pattern, subgroups are separated with a line and have significant differences $(P<.05)$ in volume-averaged $\mathrm{F}_{\mathrm{IO}_{2}}$ with other subgroups; in addition, for each breathing pattern, subgroups are arranged by magnitude of $\mathrm{F}_{\mathrm{IO}_{2}}$, in descending order; the One $\mathrm{G} 4$ does not feature device settings $>3$, and only the SimplyGo features pulse settings up to 6 .

$\mathrm{CFO}=$ continuous flow oxygen

observed among the devices when operated at the same device setting for any of the 3 breathing patterns. Although the Inogen devices delivered similar $\mathrm{F}_{\mathrm{IO}_{2}}$ as the
SimplyGo for the exercise breathing pattern, $\mathrm{F}_{\mathrm{IO}_{2}}$ was greater for the Inogen devices than for the SimplyGo devices for the rest and sleep breathing patterns. 


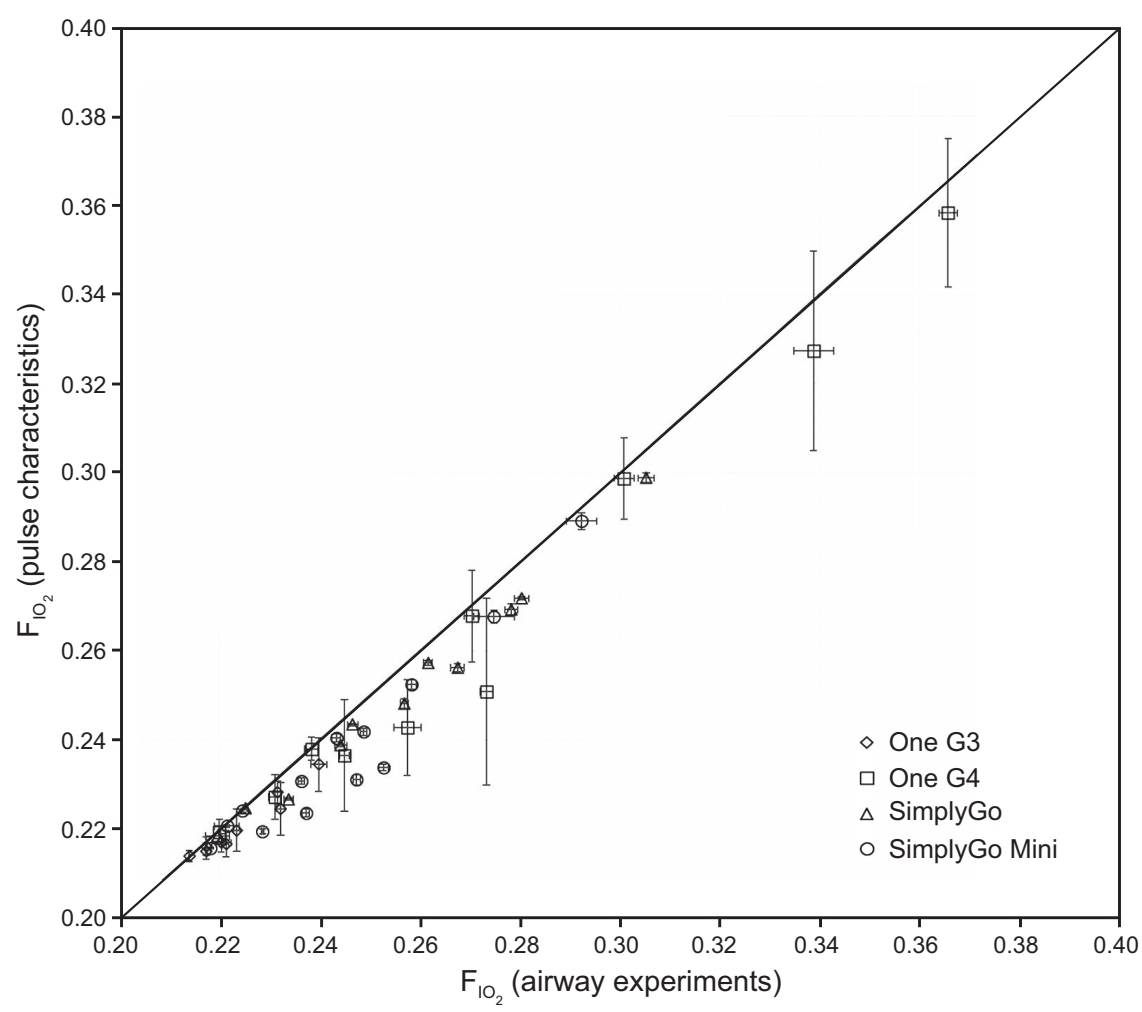

Fig. 7. Comparisons of volume-averaged $\mathrm{F}_{\mathrm{IO}_{2}}$ measured in airway experiments with volume-averaged $\mathrm{F}_{\mathrm{IO}_{2}}$ predicted by using pulse characteristics. Vertical error bars indicate $\pm 1 \mathrm{SD}$ of $\mathrm{F}_{\mathrm{IO}_{2}}$ determined from airway experiments over 5 consecutive breaths. Horizontal error bars indicate $\pm 1 \mathrm{SD}$ of $\mathrm{F}_{\mathrm{IO}_{2}}$, determined by using pulse characteristics over 20 consecutive breaths. Identity line shown for comparison.

\section{Prediction of Volume-Averaged Tracheal $\mathrm{F}_{\mathrm{IO}_{2}}$ When Using Measured Pulse Characteristics}

At a given nominal device setting, different POCs provided different oxygen volumes per breath (Fig. 5). For a given POC, pulse volume increased approximately linearly with the device setting number across each breathing pattern. In general, increasing pulse volumes were correlated with increasing values of volume-averaged $\mathrm{F}_{\mathrm{IO}_{2}}$. The longest pulse durations were observed in the SimplyGo, whereas the shortest pulse durations were observed in the SimplyGo Mini (Fig. 5). At the frequencies considered (13 to 22 breaths/min), pulse durations correlated positively with the setting number but correlated negatively with breathing frequency.

A comparison of volume-averaged tracheal $\mathrm{F}_{\mathrm{IO}_{2}}$ derived from airway experiments with those calculated from pulse characteristics when using Equation 2 are shown in Figure 7. For the sleep breathing pattern, there were no anticipated clinically important differences between the 2 sets of $\mathrm{F}_{\mathrm{IO}_{2}}$ predictions (ie, absolute difference of $<2 \%$ ) for any of the device settings in any of the devices. For the rest and exercise cases, only setting 5 of the One G3 resulted in a significant difference between the airway experiment $\mathrm{F}_{\mathrm{IO}_{2}}$ and pulse characteristics-derived $\mathrm{F}_{\mathrm{IO}_{2}}$. More generally, increasing the minute volume resulted in a higher absolute difference between the $2 \mathrm{~F}_{\mathrm{IO}_{2}}$ sets, although most of these differences did not meet the threshold for anticipated clinical importance.

\section{Mathematical Modeling}

A sample output of the mathematical model for oxygen transport in the lower airways is shown in Figure 8. The "trachea" curve is an oxygen waveform as measured in the in vitro airway replica experiments. Each subsequent curve shows the oxygen fraction waveform that varies in time as it is transported through the airways up to the terminal bronchioles (generation 14) that mark the boundary with the acini. Simulation results, which show the volume of oxygen delivered to the trachea, the volume of oxygen transported to the acini, and the ratio of acinar to tracheal volume of oxygen delivered (ie, the acinar oxygen delivery efficiency) are summarized in Figure 9.

Generally, acinar oxygen delivery efficiencies were positively correlated with increasing minute volume. Although all of the devices showed an increase in efficiency with increasing device setting in the exercise and rest cases, for 


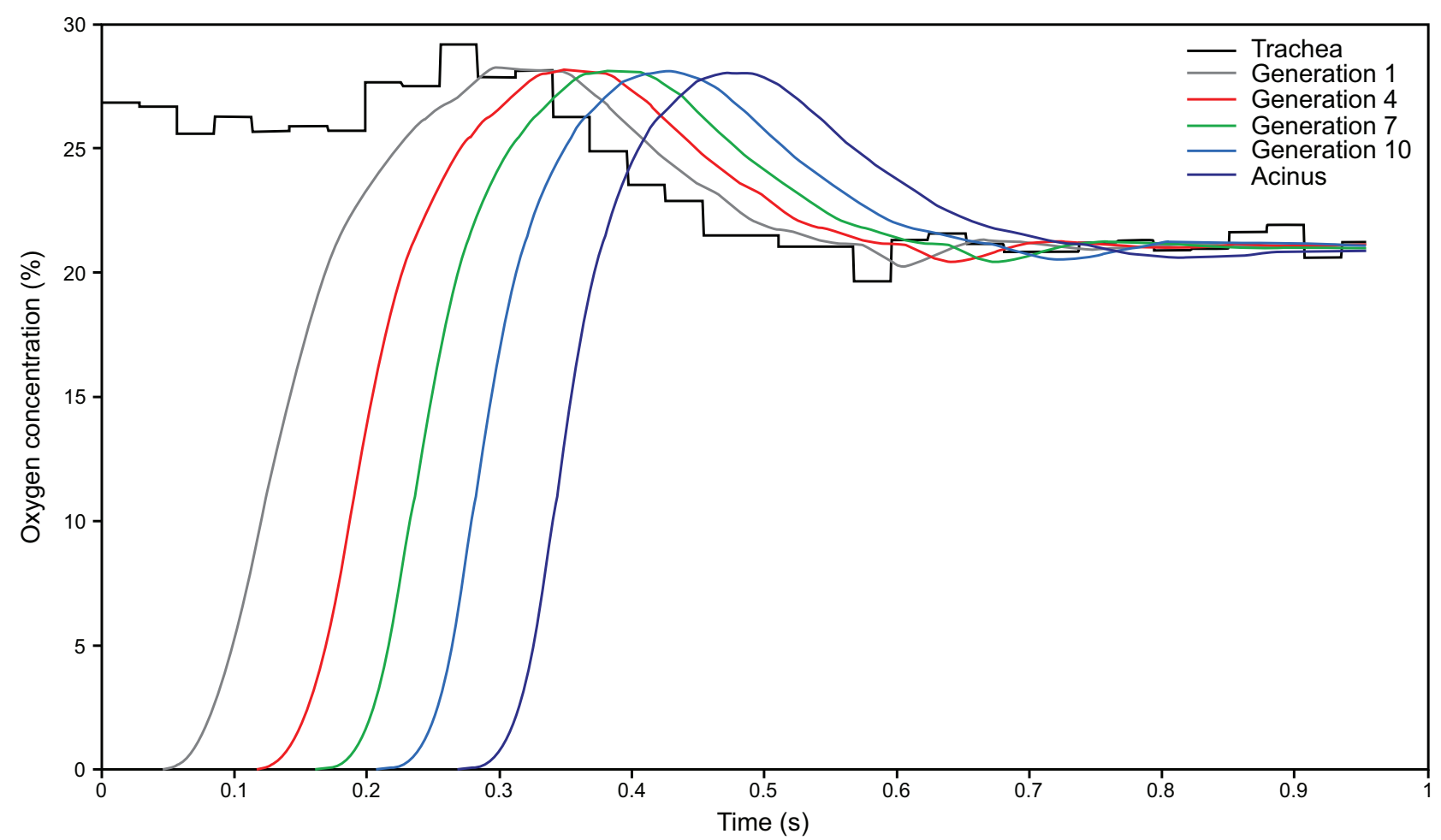

Fig. 8. Sample output of MATLAB simulation (SimplyGo Mini, exercise breathing pattern, device setting 5). The trachea line represents oxygen concentration passing the trachea over time determined by the in vitro experiments. Oxygen passing generation 14 enters the gas exchange regions of the lung.

the sleep case, the SimplyGo Mini was the only device in which this trend persisted. Efficiencies for pulse flow were generally higher than those for continuous flow oxygen. However, absolute oxygen delivery to the gas exchange region remained lower for pulse flow than for continuous flow oxygen at nominally equivalent settings and flows (Fig. 9). Differences in oxygen delivery between continuous flow oxygen and pulse flow were smaller at the acinar region than at the trachea. On average, lower minute volumes resulted in higher differences in delivered oxygen volume.

\section{Discussion}

In this study, we compared the performance of pulse flow oxygen delivery from POCs to continuous flow oxygen delivery from a stationary cylinder source. Overall, the results corroborated conclusions from previous studies ${ }^{16-18}$ in that there was no general equivalence in oxygen delivery between continuous flow oxygen in L/min and any of the nominally equivalent pulse flow settings for the POCs that we tested. At most numerical pulse settings, volume-averaged tracheal $\mathrm{F}_{\mathrm{IO}_{2}}$ was significantly lower, in both a statistical and an anticipated clinical sense, for pulsed delivery than for a nominally equivalent continuous flow oxygen flow (Fig. 5). Statistically, differences in $\mathrm{F}_{\mathrm{IO}_{2}}$ among the 4 POCs stud- ied occurred more frequently at higher device settings (Table 4), which indicated that differences in performance among the devices become more pronounced as oxygen delivery increases. This is corroborated by the fact that anticipated clinically important differences in $\mathrm{F}_{\mathrm{IO}_{2}}$ also tended to occur more frequently at higher device settings-continuous flow oxygen flows.

Large differences in pulse volumes among POCs at the same numerical device setting tended to result in large differences in volume-averaged $\mathrm{F}_{\mathrm{IO}_{2}}$. It was observed that the Inogen devices modulated pulse volumes based on breathing frequency to maintain relatively similar volumes of oxygen delivered per minute, whereas the SimplyGo and SimplyGo Mini maintained relatively constant pulse volumes for the range of frequencies considered in this study. This resulted in differences in volume-averaged $\mathrm{F}_{\mathrm{IO}_{2}}$ between, for example, the One G3 and the SimplyGo. The magnitudes of the differences in volume-averaged $\mathrm{F}_{\mathrm{IO}_{2}}$ between the One G3 and the SimplyGo are primarily the result of 2 factors: (1) slower breathing, which causes the One G3 to increase its per-breath output, and (2) shallow breathing, which causes supplemental oxygen to represent a higher fraction of the total inhaled oxygen. Because slow breathing is also associated with shallow breathing in this study, these 2 effects acted synergistically to generate large $\mathrm{F}_{\mathrm{IO}_{2}}$ differences for the sleep breathing 

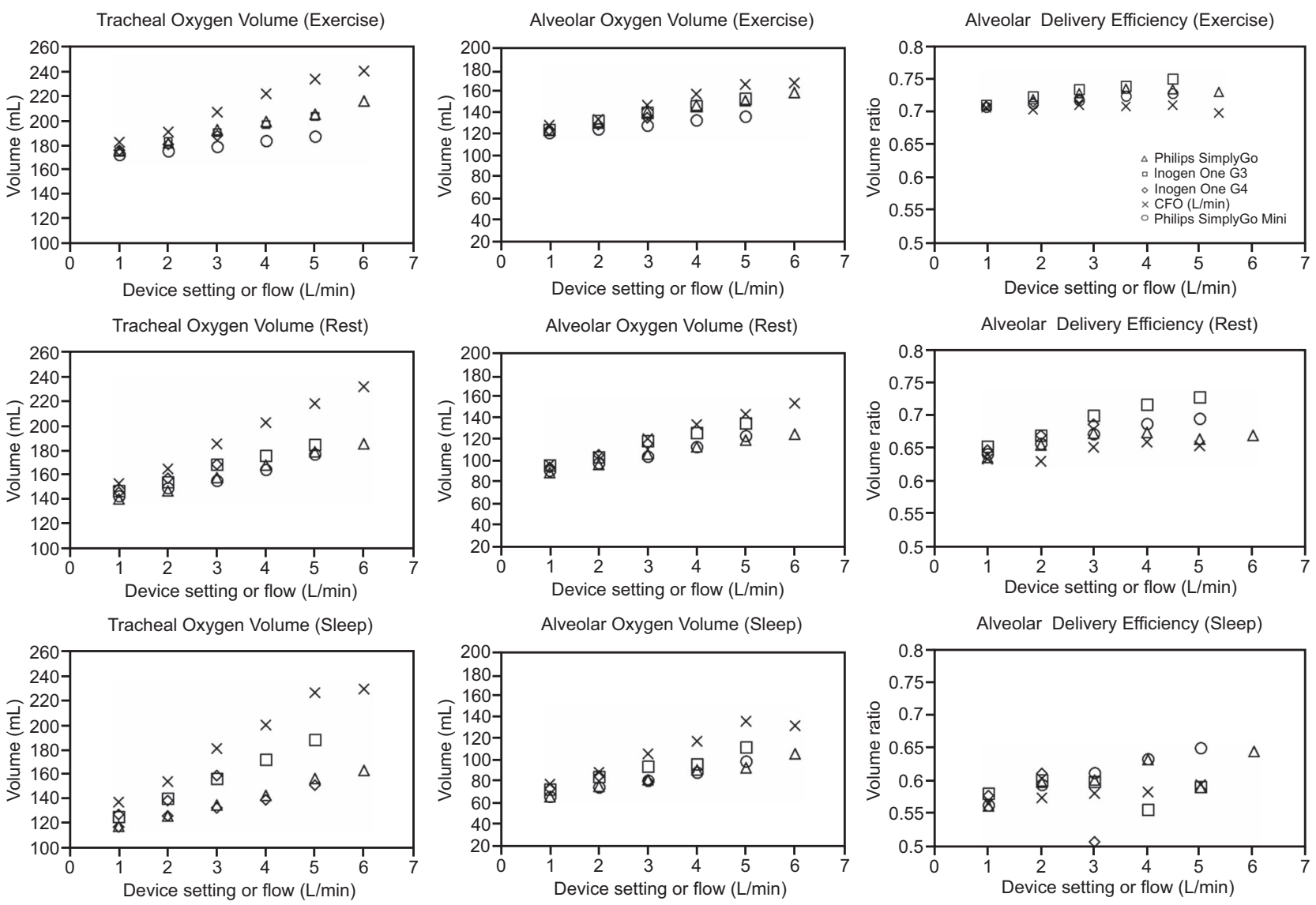

Fig. 9. Volumes of oxygen passing through the trachea, passing into the alveolar region, and the ratio of these 2 numbers (ie, a measure of the fraction of the oxygen passing the trachea that reaches the alveolar region) for all tested portable oxygen concentrators and continuous flow oxygen.

pattern (Fig. 5). Conversely, for deep, fast breathing (exercise), the $\mathrm{F}_{\mathrm{IO}_{2}}$ difference is essentially nonexistent.

Variation in measured tracheal $\mathrm{F}_{\mathrm{IO}_{2}}$ among POCs at a given setting could largely be predicted from pulse characteristics (Fig. 7). Under the present test conditions, and for the POCs tested, pulse timing had a relatively minor influence on oxygen delivery compared with pulse volume; in other words, the POCs tended to function as intended and delivered pulses early in the inspiratory phase of the breath. Although previous experiments in the literature that compared $\mathrm{F}_{\mathrm{IO}_{2}}$ between pulse flow and continuous flow oxygen modes of delivery exist, ${ }^{16,17}$ the choice in the present work to measure oxygen over time at the trachea of a realistic airway model (instead, for example, of oxygen concentration inside the test lung) is a key methodological difference from past studies. In previous studies, the conducting airways were represented by using a length of tubing of constant diameter. ${ }^{16-18}$ In reality, the tracheobronchial tree consists of a series of branching airways, with a highly variable diameter, depending on depth in the lung. ${ }^{13,19}$ Therefore, in the present work, a more complex, although still idealized, multi-generational mathematical model of the conducting airways was adopted to assess trans- port from the distal end of the trachea into the acini of the lung.

In the present mathematical model, the initial oxygen concentration in the conducting airways must be independently specified. The choice of a concentration of zero was made so to model, in absolute terms, the amount of freshly delivered oxygen passing the trachea that was transported in a single inhalation to the acini. The present combination of in vitro and in silico, or mathematical, modeling approaches inherently included the influence of pulse timing on efficiency of delivery to the acini because POCs are tested under realistic triggering conditions. Efficiency of delivery tended to be positively correlated with minute volume (Fig. 9). That is, efficiencies of the exercise breathing pattern were higher than those of the rest breathing pattern, which were, in turn, higher than those of the sleep breathing pattern. The arrival of fresh oxygen can be identified in Figure 8 as the time at which oxygen concentration increases from zero. Because the model accounts for both convection and diffusion, a greater inhalation velocity transports gas and, therefore, oxygen, more quickly, which leads to an earlier arrival time at each generation of the airway and a 


\section{Pulsed Dose vs Continuous Flow Oxygen Delivery}

higher delivery efficiency as a result. Predicted efficiency of pulse delivery to the acini (Fig. 9) still varied to some extent between POCs and POC settings for the same breathing pattern. This variability resulted from differences observed in the in vitro experiments in the timing with which pulses arrived at, and swept past, the trachea, and from differences in the volume of the oxygen pulse delivered.

Overall, pulse flow was predicted to offer advantages in efficiency of delivery, in that the fraction of oxygen delivered to the trachea that reaches gas exchange lung regions was predicted in general to be greater for pulse flow than for continuous flow oxygen (Fig. 9). This was due to the oxygen that remained in the anatomic dead space at the end of inhalation in continuous flow oxygen and never reached the gas exchange regions. But efficiency does not necessarily imply efficacy; volumes of oxygen delivered to the acini by using continuous flow oxygen were still in most cases higher than those that used pulse flow. As noted by McCoy, ${ }^{3}$ oxygen delivery must first and foremost meet the therapeutic needs of the patient. Differences in oxygen delivery reported herein between continuous flow oxygen and pulse flow settings highlight the need to titrate delivery settings to achieve a target oxygen saturation when using the same delivery device as used at home. ${ }^{2}$ Results of in vitro experiments and in silico analysis, such as those presented here, may inform this process by anticipating differences in oxygen delivery among devices and modes of administration, thereby aiding health practitioners in selecting the optimal devices for their patients.

\section{Conclusions}

In this study, in vitro experiments that used a realistic upper-airway replica were performed to compare continuous flow oxygen delivery with pulse flow from 4 commercial POCs. The volume-averaged $\mathrm{F}_{\mathrm{IO}_{2}}$ measured at the trachea was evaluated for 3 simulated breathing patterns, representative of patients with COPD at rest, during light exercise, and while asleep. $\mathrm{F}_{\mathrm{IO}_{2}}$ was not equivalent between pulse flow and continuous flow oxygen flow, and differences in oxygen delivery were greatest when high pulse flow settings were combined with low minute volume. Anticipated clinically important differences $\left(>2 \%\right.$ absolute difference in $\mathrm{F}_{\mathrm{IO}_{2}}$ ) were observed at all nominally equivalent pulse flow settings higher than 2. Significant differences in oxygen delivery were also measured among the different POCs operated at identical numerical pulse flow settings, with the clinically important differences occurring at the highest setting numbers ( 3 or 5 , depending on the device).

By coupling in vitro measurements with a mathematical model of oxygen transport through the conducting airways, it was predicted that pulse flow is generally more efficient than continuous flow oxygen at delivering oxygen from the trachea to the acini. However, acinar oxygen delivery remained lower for pulse flow than for continuous flow oxygen, at nominally equivalent settings and flow. Significant differences in oxygen delivery persisted to the acini among POCs operating at identical pulse flow settings.

\section{ACKNOWLEDGMENTS}

The authors thank Kelvin Duong at the University of Alberta for his contributions to data collection and analysis.

\section{REFERENCES}

1. Croxton TL, Bailey WC. Long-term oxygen treatment in chronic obstructive pulmonary disease: recommendations for future research: an NHLBI workshop report. Am J Respir Crit Care Med 2006; 174(4):373-378.

2. Lacasse Y, Bernard S, Maltais F. Eligibility for home oxygen programs and funding across Canada. Can Respir J 2015;22(6):324-330.

3. McCoy R. Oxygen-conserving techniques and devices. Respir Care 2000;45(1):95-103; discussion 104.

4. Bliss PL, McCoy RW, Adams AB. Characteristics of demand oxygen delivery systems: maximum output and setting recommendations. Respir Care 2004;49(2):160-165.

5. Palwai A, Skowronski M, Coreno A, Drummond C, McFadden ER Jr. Critical comparisons of the clinical performance of oxygen-conserving devices. Am J Respir Crit Care Med 2010;181(10):1061-1071.

6. Tiep B. Portable oxygen therapy with oxygen conserving devices and methodologies. Monaldi Arch Chest Dis 1995;50(1):51-57.

7. Airsep Freestyle Patient Manual. MN123-1 rev E 11/12. Buffalo, NY: AirSep.

8. Yáñez AM, Prat JP, Álvarez-Sala JL, Calle M, Diaz Lobato S, Garcia Gonzalez JL, et al. Oxygenation with a single portable pulse-dose oxygen-conserving device and combined stationary and portable oxygen delivery devices in subjects with COPD. Respir Care 2015; 60(3):382-387.

9. Wijkstra PJ, Guyatt GH, Ambrosino N, Celli BR, Güell R, Muir JF, et al. International approaches to the prescription of long-term oxygen therapy. Eur Respir J 2001;18(6):909-913.

10. Chen JZ, Katz IM, Pichelin M, Zhu K, Caillibotte G, Noga ML, et al. Comparison of pulsed versus continuous oxygen delivery using realistic adult nasal airway replicas. Int J Chron Obstruct Pulmon Dis 2017;12:2559-2571.

11. Chatila W, Nugent T, Vance G, Gaughan J, Criner GJ. The effects of high-flow vs low-flow oxygen on exercise in advanced obstructive airways disease. Chest 2004;126(4):1108-1115.

12. Hudgel DW, Martin RJ, Capehart M, Johnson B, Hill P. Contribution of hypoventilation to sleep oxygen desaturation in chronic obstructive pulmonary disease. J Appl Physiol Respir Environ Exerc Physiol 1983;55(3):669-677.

13. Martin AR, Jackson C, Katz IM, Caillibotte G. Variability in uptake efficiency for pulsed versus constant concentration delivery of inhaled nitric oxide. Med Gas Res 2014;4(1):1.

14. Finlay WH, Lange CF, King M, Speert DP. Lung delivery of aerosolized dextran. Am J Respir Crit Care Med 2000;161(1):91-97.

15. Phillips CG, Kaye SR, Schroter RC. A diameter-based reconstruction of the branching pattern of the human bronchial tree. Part I. Description and application. Respir Physiol 1994;98(2): 193-217.

16. Zhou S, Chatburn RL. Effect of the anatomic reservoir on low-flow oxygen delivery via nasal cannula: constant flow versus pulse flow with portable oxygen concentrator. Respir Care 2014;59(8):1199-1209. 


\section{Pulsed Dose vs Continuous Flow Oxygen Delivery}

17. Chatburn RL, Williams TJ. Performance comparison of 4 portable oxygen concentrators. Respir Care 2010;55(4):433-442.

18. Bliss PL, McCoy RW, Adams AB. A bench study comparison of demand oxygen delivery systems and continuous flow oxygen. Respir Care 1999;44(8):925-931.

19. Finlay WH. The mechanics of inhaled pharmaceutical aerosols: an introduction. San Diego: Academic Press; 2001:151-154.
20. Agustí AG, Barberá JA, Roca J, Wagner PD, Guitart R, RodriguezRoisin R. Hypoxic pulmonary vasoconstriction and gas exchange during exercise in chronic obstructive pulmonary disease. Chest 1990; 97(2):268-275.

21. Katz IM, Martin AR, Caillibotte G, Arpentinier P. Property value estimation for inhaled therapeutic binary gas mixtures: $\mathrm{He}, \mathrm{Xe}, \mathrm{N} 2 \mathrm{O}$, and N2 with O2. Med Gas Res 2011;1:28.

This article is approved for Continuing Respiratory Care Education credit. For information and to obtain your CRCE

(free to AARC members) visit

www.rcjournal.com 